\title{
Research Paper: The Effects of 12-Weeks of Senso- rimotor Exercise on Pain, Strength, Pelvic Drop, and Dynamic Knee Valgus in Males With Patellofemoral Pain Syndrome
}

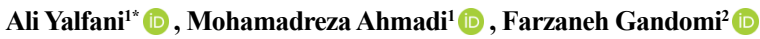 \\ 1. Department of Corrective Exercises and Sports Injury, Faculty of Physical Education and Sport Sciences, Bu Ali Sina University, Hamedan, Iran. \\ 2. Department of Corrective Exercises and Sports Injury, Faculty of Physical Education and Sport Sciences, Razi University, Kermanshah, Iran.
}

\begin{tabular}{|c|c|}
\hline $\begin{array}{l}\text { Usseyur devive to san } \\
\text { and read he artic online }\end{array}$ & \\
\hline 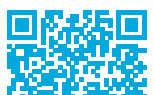 & $\begin{array}{l}\text { Drop, and Dynamic Knee Valgus in Males With Patellofemoral Pain Syndrome. Physical Treatments. 2020; 10(3):159-168. } \\
\text { http://dx.doi.org/10.32598/ptj.10.3.442.1 }\end{array}$ \\
\hline 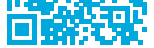 & doi): http://dx.doi.org/10.32598/ptj.10.3.442.1 \\
\hline
\end{tabular}

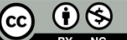

Article info:

Received: 06 Jan 2020

Accepted: 07 Jun 2020

Available Online: 01 Jul 2020

Keywords:

Patellofemoral pain syndrome, Kinematics, Pelvis, Muscle strength, Exercise

\begin{abstract}
A B S T RA C T
Purpose: Patellofemoral Pain Syndrome (PFPS) is among the most prevalent complaints observed in healthcare clinics. This group of patients encounters Pelvic Drop (PD) and Dynamic Knee Valgus (DNV); its' causes have been reported as pain and decreased muscle strength. The current study aimed to explore the effects of a 12-week Sensorimotor Exercise (SME) on pain, strength, PD, and DNV in males with PFPS.
\end{abstract}

Methods: This randomized double-blind clinical trial involved 32 patients with PFPS. The study samples were randomly divided into the experimental $(\mathrm{n}=16)$ and control $(\mathrm{n}=16)$ groups. To assess pain, the Visual Analogue Scale (VAS) was used. Moreover, quadriceps muscle strength was measured by the hip abductor hand dynamometer. To analyze PD and DNV, camera and Kinova software were used during stairs descent. The experimental group performed 12 weeks of SME for 3 one-hour weekly sessions. However, the control group received no therapeutic intervention during this time. The obtained data were analyzed in SPSS using Analysis of Covariance (ANCOVA).

Results: The data analysis results suggested that pain significantly reduced in the experimental group, compared to the control group, after twelve weeks of $\operatorname{SME}(\mathrm{P}<0.001)$. Besides, quadriceps muscle strength $(\mathrm{P}<0.002)$ and hip abductor muscle strength $(\mathrm{P}<0.001)$ improved, and $\mathrm{PD}$ angle $(\mathrm{P}<0.002)$ and $\mathrm{DNV}(\mathrm{P}<0.003)$ were reduced.

Conclusion: SME reduced pain and facilitated the frequency and time of muscle activation. It also increased the strength of the gluteus medius muscle as the main stabilizer of the pelvis chain and led to a reduction in PD and DNV. Thus, SME could be used as a comprehensive protocol treatment to improve various disorders in patients with PFPS.

\footnotetext{
* Corresponding Author:

Ali Yalfani, $P h D$.

Address: Department of Corrective Exercises and Sports Injury, Faculty of Physical Education and Sport Sciences, Bu Ali Sina University, Hamedan, Iran. Phone: +98 (918) 3155478

E-mail:ali_yalfani@yahoo.com
} 


\section{Highlights}

- SME reduced pain and increased strength, facilitated the frequency and activation time of quadriceps and hip abductor muscles.

- SME reduced PD and DNV.

\section{Plain Language Summary}

Therapists are always looking for a comprehensive treatment approach to provide the best therapeutic outcome with an emphasis on improving movement patterns in patients with PFPS. The present study provided a comprehensive treatment approach for patients with PFPS.

\section{Introduction}

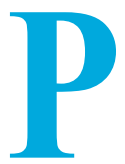

atellofemoral Pain Syndrome (PFPS) is described as anterior or retropatellar knee pain. It is also among the most common complaints observed in healthcare centers. The outbreak of this disease is more prevalent in active young individuals aged under 50 years

$[1,2]$. Several factors have been suggested to explain the presence of PFPS. These characteristics include decreased muscle strength and altered lower limb biomechanical and muscle activation patterns [2].

Biomechanical factors reported in the development and occurrence of PFPS is malignant lower limbs; it is introduced among the most frequent disorders of malignant of the lower limbs of Dynamic Knee Valgus (DNV) and Pelvic Drop (PD) [2-4]. In explaining this theory, it can be referred to studies by Staulo et al. [3] and Neal et al. [4] that reported an increase in DNV and PD during functional movements, in comparison to healthy individuals.

The increasing DNV of the patella external traction follows enhanced contact pressure on the external patella with the external condyle thigh; it could damage the joint cartilage behind the patella and cause or aggravate pain [5]. Controlling DNV in the frontal plane is critical during dynamic activities. This is because it is associated with increased stress on the passive structure and abnormal distribution of load around the Patellofemoral Joint (PFJ). Subsequently, it results in the development of destructive processes or the occurrence of PFPS [6]. It could also affect the proximal segmental; accordingly, in the proximal part, it causes weak neuromuscular control of proximal factors or weakness in the hip muscles [7]. However, the main mechanism of DNV and PD in patients with PFPS remains unclear. Factors, such as weakness in the hip abductor muscle strength, especially the Gluteus Medius (GM), and experiencing pain have been reported in this regard $[5,8]$. Accordingly, not understanding the mechanism of the occurrence of PD and DNV and developing comprehensive treatment protocols are challenging issues for researchers and rehabilitation specialists. Moreover, there is little research in this area; therefore, understanding the root cause and designing a comprehensive treatment protocol could significantly affect the prevention and reduction of PD and DNV, i.e. among the main factors in PFPS occurrence [3].

Sensory-Motor Exercises (SME) provided by Janda are designed to manage pain in patients with chronic musculoskeletal pain syndrome. These exercises could also correct muscle imbalance and movement program at the Central Nervous System (CNS) level; by reproducing neuromuscular functional adaptability and reducing the prevalence of injuries, proprioception, and intramuscular coordination are improved [9].

Studies are available on the effectiveness of SME in controlling musculoskeletal pain syndromes; however, no research investigated the effects of this exercise in managing pain, as well as improving strength, $\mathrm{PD}$, and DNV in patients with PFPS. Such investigations could provide a comprehensive treatment approach for these patients. Therefore, this study aimed to explore the following hypotheses: Twelve weeks of SME leads to pain relief; 12 weeks of SME leads to an increase in strength of the quadriceps and hip abductor muscles, and 12 weeks of SME leads to a reduction in the DNV and PD angle.

\section{Materials and Methods}

The present randomized double-blind clinical trial included men with PFPS who referred to orthopedic clinics in Hamadan Province, Iran. The study participants were divided into two groups of exercise therapy $(n=16)$ and control $(n=16)$. All tests were performed by laboratory specialists in the 
sports rehabilitation laboratory of Bu Ali Sina University of Hamadan, Iran. Before the patients entered the laboratory, the researcher provided the patient with information about the evaluation process of the present study. After all of the patients were informed about the study process, they joined the present study by signing an informed consent form. This project was approved by and registered at the National Ethics Committee in Biomedical Research (code: IR.BASU.1398.001) and the clinical trial center (code: IRCT 20191209045669N1). Besides, it was conducted as per the 2008 Helsinki Declaration.

The statistical population of the present study included male patients with PFPS in the age range of 18 to 35 years. For screening patients, the step-down test (ICC:0.94) was used [10]; the patient placed his hands on his chest and descended from a step to a height of $30 \mathrm{~cm}$ in a controlled manner. This movement was repeated 5 to 10 times and the increase in the pain intensity and discomfort indicated the positive result of the test [11]. To estimate the sample size, G*Power was used, the values applied in the software are per a previous study (power $=0.80$, effect size $=0.25, \alpha=0.05$ ) [12].

The study sample consisted of 32 patients ( $n=16 /$ group) who participated in the study base on the inclusion and exclusion criteria. The study inclusion criteria included the following: experiencing pain around PFJ for more than 6 weeks, increased pain intensity in the anterior or posterior part of the patella at least by one of the activities as long-time sitting, kneeling, running, squatting, jumping, and ascent and descent stairs [13]. The study exclusion criteria included the following: injury and hip pain, lumbar spine, other knee joint structures, such patella tendon, surgical history, neurological disorders, PFJ instability, knee joint effusion, receiving physiotherapy in the previous year to treat PFPS, lower limb deformity or weekly use of anti-inflammatory medications [13]. After completing the assessments, all explored patients were homogenized based on age, height, weight, Body Mass Index (BMI), pain, strength, PD, and DNV were randomized by Random Number Generator software; they were then divided into the experimental and control groups based on the SNOSE method. The first assessment stage concerned demographic information indices, including age, height, weight, and BMI. These characteristics were measured using a digital scale.

The second evaluation stage included measuring the lower limb length. At this stage, the patient lied on his back on the examination table and the distance between the anterior superior iliac spine up to malleus internal was recorded as the lower limb length. This evaluation was performed for both limbs [14].
The third stage was related to the measurement of PD and $\mathrm{DNV}$, i.e. performed in the form of two dimensions when descending 7 steps with a height of $18 \mathrm{~cm}$ and a width of $28 \mathrm{~cm}$ [15]. To evaluate the movement pattern, a Sony Handycam DCR-HC37 digital camera (made in Japan) with a sampling rate of $40 \mathrm{~Hz}$ and a 10 optical zoom was employed. It was calibrated by laboratory experts during the evaluation of each attempt. The first camera was set at a distance of 3 meters from the stairs parallel to the frontal plane and per the knee height. Furthermore, the second camera was set at the same height and distance from the stairs and parallel to the sagittal plane [5]. Four markers were then attached in the following segments: the anterior superior iliac spine on both sides, the center of the patella, and the center of the ankle joint in the limb that could perform landing [16]. Before evaluating the movement pattern, the patients performed a three-step descent to become familiar with the method. They were also instructed to perform the movement at a normal velocity, like daily living activities [17].

The DNV and PD angles were assessed while the patient staired descend without using a railing while placing both hands crosswise on the chest; PD and DNV were calculated by Kinova Software $(\mathrm{ICC}=0.97)[18]$ when the knee was in the fourth step at its peak flexion [8]. As a result, the value obtained from the DNV fell below 180 and it was used in the final analysis [5]. The DP was evaluated by determining the angle between the line that connects the anterior superior iliac spine on both sides and the line perpendicular to the upper anterior iliac spine of the limb landing [16]. The obtained value was then subtracted from 90 and recorded as the PD angle [16]. Additionally, the movement pattern of descending the stairs was performed 5 times and the average score of 5 attempts was calculated as the final angle [8]. The studied patients were also requested to report their pain intensity as their stairs descended. A $10-\mathrm{cm}$ visual analog scale $(\mathrm{ICC}=0.91)$ was implemented to measure pain intensity [19]; the reliability of this scale has been reported to range from $77 \%$ to $79 \%$ for patients with PFPS [20].

The fourth assessment stage concerned measuring muscle stre ngth by a hand-held dynamometer (Nicholas HHD; 01163 model of 'Lafayette instrument, made in England) (ICC $=0.89-0.94$ ) [21]. The calibration of the HHD was established before the study by placing a specific weight on the HHD and comparing it with the weight indicated by it [22]. Before running the experiment, the study patients performed two sub-maximal contractions to become familiarized with the method [14].

The method for quadriceps muscle was as follow: the patient sat on the examination table with the hip and knees at a $90^{\circ}$ flexion [23] and hands were held crosswise 
upon chest; then, the tester placed the HHD in the front and between two malleoli and the patient performed the maximum isometric contraction [23]. To assess hip abductor muscle strength, the patient was placed in a sidelying position on the treatment table with the testing limb on top. The examiner checked the limb to assure the lack of external rotations or extensions and placed a pillow between the legs to neutralize the hip position [14]. The tester placed HHD on the lateral femoral condyle and the patient performed the maximum isometric contraction [23]. Each contraction was conducted for 5 seconds and 3 times with the average value recorded. Besides, a 2-minute interval was considered between the trials [14].

SME was performed three times a week for 12 onehour weekly sessions ( 5 minutes of warm-up +50 minutes of exercise +5 minutes of cool-down) by the experimental group. The control group received no treatment program and did not use analgesics. SME was selected based on a previous study [24] and all designed movements were extracted from valid studies and sources [2428]. This treatment protocol included three stages; static, dynamic, and functional. Furthermore, it was developed by challenging posture, the center of gravity, and supporting surface [24]. Before each exercise, the rehabilitation specialist theoretically and practically instructed the patient to perform the movement correctly; then, the patients performed the selected exercise. Each exercise lasted from 5 to 20 seconds; the number of repetitions for hard exercises was 5, and for easy exercises, it took up to 20 repetitions [24]. Table 1 presents the treatment protocol.

For statistical analysis, SPSS was used. The levels of significance and confidence for analyzing all data were considered $t$ be 0.5 and $95 \%$, respectively. To ensure the normality

Table 1. Sensory-Motor training protocol

\begin{tabular}{|c|c|}
\hline Stage & Exercise \\
\hline $\begin{array}{c}\text { Static } \\
\text { (weeks } 1 \text { to 4) }\end{array}$ & $\begin{array}{l}\text { - Warm-up } \\
\text { - Standing on two legs (Romberg) on a hard surface with eyes open (with \& without disturbance) } \\
\text { - Standing on both legs on a soft surface (Romberg) with eyes closed (with \& without disturbance) } \\
\text { - Marching standing with eyes open on a hard surface (with \& without disturbance) } \\
\text { - Marching standing with eyes closed on a soft surface (with \& without disturbance) } \\
\text { - Standing tandem on a hard surface with eyes open (with \&without disturbance) } \\
\text { - Standing tandem on a soft surface with eyes closed (with \& without disturbance) } \\
\text { - Static mini-squat on a hard surface with eyes open (with \& without disturbance) } \\
\text { - Static mini-squat on a soft surface with eyes closed (with \& without disturbance)Cool-down }\end{array}$ \\
\hline $\begin{array}{c}\text { Dynamics } \\
\text { (weeks } 5 \text { to 8) }\end{array}$ & $\begin{array}{l}\text { - Warm-up } \\
\text { - Half a step on an unstable surface } \\
\text { - Shooting in front of Traband on a soft surface in the directions of internal, external, posterior, anterior } \\
\text { - Stand on one leg on a hard surface by catching and throwing the ball } \\
\text { - Stand on one leg on a soft surface by catching and throwing the ball } \\
\text { - Launch forward in a fixed position (progress: add resistance band) } \\
\text { - Launch to the side in a fixed position (progress: add resistance band) } \\
\text { - One-legged marching stand on a hard surface in front of Traband } \\
\text { - One-legged marching stand on a soft surface in front of TrabandCool-down }\end{array}$ \\
\hline Functional (weeks 9 to 12 ) & $\begin{array}{l}\text { - Warm-up } \\
\text { - Walk backward with eyes open } \\
\text { - Squat leans back against the wall } \\
\text { - Squat on the unstable surface of the double-legged } \\
\text { - Launch forward with weights } \\
\text { - Ascent and descent stairs (front \& back) } \\
\text { - Ascent and descent stairs (side) } \\
\text { - Single-legged jump on the frontal plane against the Traband } \\
\text { - Single-legged jump on the frontal plate against Traband along with catching and throwing the ball Cool-down }\end{array}$ \\
\hline
\end{tabular}


Table 2. Demographic characteristics of the study patients and Shapiro-Wilk' test results

\begin{tabular}{|c|c|c|c|}
\hline \multirow{2}{*}{ Variable } & \multicolumn{2}{|c|}{ Mean $\pm S D$} & \multirow{2}{*}{$\mathbf{P}$} \\
\hline & Experimental Group & Control Group & \\
\hline Age, y & $25.60 \pm 2.35$ & $25.00 \pm 1.93$ & 0.181 \\
\hline Height, $\mathrm{cm}$ & $173.40 \pm 3.81$ & $174.93 \pm 4.09$ & 0.063 \\
\hline Weight, kg & $73.40 \pm 5.76$ & $74.33 \pm 6.43$ & 0.296 \\
\hline $\mathrm{BMI}, \mathrm{kg} / \mathrm{m}^{2}$ & $24.44 \pm 1.67$ & $24.26 \pm 1.34$ & 0.871 \\
\hline Lower limb length, $\mathrm{cm}$ & $89.96 \pm 2.47$ & $90.66 \pm 2.25$ & 0.491 \\
\hline
\end{tabular}

PHYSICAL TREA TMENTS

of data related to variables and demographic information, Shapiro-Wilk's test was used. Levene's test was also employed for testing the homogeneity of variances. Moreover, to investigate the before and after values of the 12-week SME, Analysis of Covariance (ANCOVA) was used.

\section{Results}

In total, 32 patients with PFPS ( $\mathrm{n}=16$ patients per experimental \& control groups) participated in the present study. The Mean \pm SD age of them was $25.30 \pm 2.13$ years; their Mean \pm SD height was $174.16 \pm 3.96 \mathrm{~cm}$, their Mean \pm SD weight was $73.86 \pm 6.02 \mathrm{~kg}$, their Mean \pm SD BMI was $24.35 \pm 1.49 \mathrm{~kg} / \mathrm{m}^{2}$, and their lower limb length equaled $90.31 \pm 2.35 \mathrm{~cm}$. Table 2 lists the distribution of patients' demographic characteristics, i.e. statistically normal $(\mathrm{P}=0.05)$. Thus, parametric tests were used to check the hypotheses. Table 3 demonstrates the ANCOVA results. The obtained results suggested that performing 12 weeks of SME significantly reduced pain in the experimental group $(\mathrm{P}<0.001)$, improved quadriceps muscle strength $(\mathrm{P}<0.002)$, improved hip abductor muscle strength $(\mathrm{P}<0.001)$, and reduced $\mathrm{PD}(\mathrm{P}<0.002)$ as well as DNV angle $(\mathrm{P}<0.003)$.

\section{Discussion}

The current study evaluated the effects of 12 weeks of SME on pain, strength, PD, and DNV in patients with PFPS. The collected results indicated that the provided intervention significantly reduced pain, improved quadriceps, and hip abductor muscle strength, and reduced PD and DNV in the experimental group, compared to the controls. These findings supported all of our hypotheses; accordingly, the mechanism of effectiveness of the treatment protocol on each variable is discussed.

The achieved results indicated that the explored patients' pain intensity was decreased after conducting 12 weeks of SME; thus, it supports our first hypothesis and is consistent with the study of Ahmed et al. [26] and inconsistent with the study of McCaskey et al. [29]; this inconsistency may be due to the short duration of the intervention (4 weeks). Pain relief could be related to two mechanisms The firs: the mechanism is due to muscle balance created after SME in the vastus medialis and lateralis muscles. Accordingly, it leads to the normal position of the patella inside the condylar groove of the thigh; thus, relieving pain and improving neuromuscular control [30]. The second mechanism is due to the increase in the strength and proper activation of the GM; it overcomes the compensa-

Table 3. ANCOVA results

\begin{tabular}{|c|c|c|c|c|}
\hline Variable & $\mathbf{F}$ & Power & Eta Squared & $\mathbf{P}^{*}$ \\
\hline Pain & 80.580 & 1 & 0.749 & 0.001 \\
\hline Quadriceps muscle strength & 79.178 & 1 & 0.746 & 0.002 \\
\hline $\begin{array}{l}\text { Hip abductor muscles } \\
\text { Strength }\end{array}$ & 47.650 & 1 & 0.638 & 0.001 \\
\hline Pelvic drop & 11.355 & 0.901 & 0.296 & 0.002 \\
\hline Dynamic knee valgus & 10.844 & 0.888 & 0.287 & 0.003 \\
\hline
\end{tabular}

* Significant level PHYSICAL TREA TMENTS 
tory function of the iliotibial band and retinal culum external and prevents external patella traction [31].

There was also an improvement in quadriceps and hip abductor muscle strength. This result supported our second hypothesis, i.e. consistent with the study of Ahmed et al. [25], and inconsistent with the study of Bruhn et al. [32]; this finding may be due to the health of individuals. The effectiveness mechanism of the treatment protocol on muscle strength could be attributed to several factors, as explained in the following: The first factor could be due to the effect of adaptation created in the CNS; during the exercises, learning and adapting the stimulation pattern were increased. Ultimately, it enhanced maximal voluntary contraction [24].

The second factor included increasing sensory input about joint position and changes in muscle length and tension in the CNS. In turn, it improves the nervous system's ability to generate a pattern of rapid and optimal muscle utilization and increases the number of active motor units. Moreover, increased intragroup and intergroup muscle coordination leads to improved force generation ability [33].

The third characteristic, through dynamic stability exercises in the dynamic and functional phase and rapid muscle contraction, could improve the order and synergistic degree of contraction. Finally, muscle strength was improved; as a result, the stimulation of the CNS is the key to an initial increase in strength, especially when coordination and stability are considered [34].

The obtained data demonstrated that the rates of PD and DNV decreased after performing 12 weeks of SME. These data support our third hypotheses, i.e. consistent with the study of Amanda and colleagues [35]. The pelvis is located in the center of the movement chain in all movements [15]. Based on the movement chain theory, orthopedic or deformity conditions are extended to other sections and affect other parts and cause increased DNV and applied torque on the knee joint. Besides, it is among the biomechanical and clinical signs of PFPS [3]. Therefore, pelvic stability and the proper activation of the pelvis stabilizing muscles are necessary to control the torques applied to the segments and to maximize the correct movement pattern of the lower limbs [5]. According to previous studies, one of the most frequent causes of the mechanism of occurrence of DNV and PD is disturbances in the pattern of the activity of hip abductor muscles, especially GM muscle, and weak neuromuscular control [7, 36, 37].
In the stance phase, the force of gravity moves the pelvis to the addiction position; subsequently, at this point, the muscles' GM, gluteus minimus, and the upper part of the gluteus maximus of the same side are eccentrically contracted to counteract the varus torque and stabilize the pelvis, thus preventing PD [31]. The PD of the opposite side, i.e. due to the inefficiency of the abductor's muscles caused by a delay in the activation or reduction of muscle strength, causes the ground reaction force. Accordingly, the center of mass of the body moves towards the supporting limb [31]. As a result, the need to activate the hip muscles is reduced, which in turn leads to enhanced abduction torque and DNV [37]. Therefore, rehabilitation interventions should aim to facilitate the activation of the GM muscle to reduce PD; it ultimately leads to a correct movement pattern and reduced abnormal repetitive stresses exerted on the knee joint due to pelvis compensatory movements. Eventually, this process leads to the treatment and prevention of PFPS [37]

Accordingly, traditional therapeutic interventions in PFPS patients are mainly strengthening exercises [38, 39]; however, Palmer et al. [40] and Ferber et al. [41] found that strengthening exercises (despite increasing the production of force in the hip abductor muscles) presented no significant effect on the movement pattern and DNV. According to previous studies, in addition to weakness in muscle strength, there is an impairment in the order of proper muscle discharge in patients with PFPS, including delay in the activation and short duration of GM activity [14]; thus, the present study intervention focused on proper muscle discharge. To improve the proper activation of this group of muscle exercises that aim to automate more complex synergies was used. They included synergies that involved multiple joints, muscles, and motor levels [29].

Rehabilitation interventions focusing on the SM approach include exercises in various positions and functional movements; such training protocols enable the motor-sensory system to create unconscious motor programs to ensure the stability of the movement chain during functional activities [23, 42]. Unconscious movement programs are at the level of the spinal cord. These exercises include isolated spinal reflexes, i.e. directly influenced by afferent information from joint receptors; spinal surface reflexes are very rapid, involuntary, and unconscious and coordinate agonist and antagonist muscles $[23,43]$. With the progress of the training stages from the static stage to the dynamic and functional stages, the sequence of muscle activation, simultaneous muscle activation, and the movement control of feedforward and feedback is facilitated $[25,43]$. 
Feedforward activity contributes to the dynamic inhibition system through several factors; thus, by increasing the muscle activation level, the stiffness characteristics of all muscle-tendon units are increased. Subsequently, it improves the tensile sensitivity of the spindle system and reduces electromechanical latency. Ultimately, stiffness and tensile sensitivity are increased by providing more sensory feedback on the transmitted motor efferent. Therefore, it improves the reactivity of the activated muscles which responds quickly to external forces and leads to dynamic joint stability [43, 44].

The study limitations included small sample size, overlooking the evaluation of kinetic factors, such as ground reaction force, electromyographic activity, and loading rate, and the lack of controlling the speed during stair descent. It is suggested that future research evaluate the effect of this treatment protocol on different kinetic factors at different speeds during stair descent in large samples of PFPS patients.

\section{Conclusion}

Twelve weeks of SME seemed to significantly reduce pain, improve strength, reduce $\mathrm{PD}$, and DNV in patients with PFPS. In addition to reducing pain, SME by facilitating the frequency and time of muscle activation and increasing the strength of the GM muscle as the most important stabilizer of the pelvis chain deducted PD and DNV. As a result, SME with emphasis on the central and peripheral structures could be a comprehensive protocol for treating various conditions in patients with PFPS.

\section{Ethical Considerations}

\section{Compliance with ethical guidelines}

This project was approved by and registered at the National Committee for Ethics in Biomedical Research (IR.BASU.1398.001) and Clinical Trial Center (IRCT 20191209045669N1).

\section{Funding}

The present paper was extracted from the MA. thesis or extracted from second author, Department of Corrective Exercises and Sports Injury, Faculty of Physical Education and Sport Sciences, Bu Ali Sina University, Hamedan.

\section{Authors' contributions}

All authors contributed in preparing this article.

\section{Conflict of interest}

There are no conflicts of interest in this study to be declared.

\section{Acknowledgments}

Researchers would like to thank all the participants in this study for their cooperation with the research team that made this study possible. We would also like to thank the head of the Sports Rehabilitation Laboratory of Bu Ali Sina University in Hamadan for providing the laboratory and treatment conditions for this research and the laboratory specialists who helped us evaluate the studied patients.

\section{References}

[1] Bair MJ, Robinson RL, Katon W, Kroenke K. Depression and pain comorbidity: A literature review. Archives of Internal Medicine. 2003; 163(20):2433-45. [DOI:10.1001/ archinte.163.20.2433] [PMID]

[2] Esculier JF, Roy JS, Bouyer LJ. Lower limb control and strength in runners with and without patellofemoral pain syndrome. Gait \& Posture. 2015; 41(3):813-9. [DOI:10.1016/j. gaitpost.2015.02.020] [PMID]

[3] Di Staulo AM, Scholtes SA, Salsich GB. A descriptive report of the variability in 3D hip and knee kinematics during a single limb squat in women who have patellofemoral pain and visually classified dynamic knee valgus. Physiotherapy Theory and Practice. 2019; 1-10. [DOI:10.1080/09593985.201 9.1698082] [PMID]

[4] Neal BS, Barton CJ, Gallie R, O'Halloran P, Morrissey D. Runners with patellofemoral pain have altered biomechanics which targeted interventions can modify: A systematic review and meta-analysis. Gait \& Posture. 2016; 45:69-82 [DOI:10.1016/j.gaitpost.2015.11.018] [PMID]

[5] Gwynne CR, Curran SA. Two-dimensional frontal plane projection angle can identify subgroups of patellofemoral pain patients who demonstrate dynamic knee valgus. Clinical Biomechanics. 2018; 58:44-8. [DOI:10.1016/j.clinbiomech.2018.06.021] [PMID]

[6] Robinson RL, Nee RJ. Analysis of hip strength in females seeking physical therapy treatment for unilateral patellofemoral pain syndrome. Journal of Orthopaedic \& Sports Physical Therapy. 2007; 37(5):232-8. [DOI:10.2519/ jospt.2007.2439] [PMID]

[7] Glaviano NR, Saliba S. Association of altered frontal plane kinematics and physical activity levels in females with patellofemoral pain. Gait \& Posture. 2018; (65):86-8. [DOI:10.1016/j.gaitpost.2018.07.164] [PMID]

[8] Waiteman MC, Briani RV, Pazzinatto MF, Ferreira AS, Ferrari D, de Oliveira Silva D, et al. Relationship between knee abduction moment with patellofemoral joint reaction force, stress and self-reported pain during stair descent in wom- 
en with patellofemoral pain. Clinical Biomechanics. 2018; 59:110-6. [DOI:10.1016/j.clinbiomech.2018.09.012] [PMID]

[9] Page P. Sensorimotor training: A "global" approach for balance training. Journal of Bodywork and Movement Therapies. 2006; 10(1):77-84. [DOI:10.1016/j.jbmt.2005.04.006]

[10] Piva SR, Fitzgerald K, Irrgang JJ, Jones S, Hando BR, Browder DA, et al. Reliability of measures of impairments associated with patellofemoral pain syndrome. BMC Musculoskeletal Disorders. 2006; 7(1):33. [DOI:10.1186/14712474-7-33] [PMID] [PMCID]

[11] Petersen W, Rembitzki I, Liebau C. Patellofemoral pain in athletes. Open Access Journal of Sports Medicine. 2017 8:143-154. [DOI:10.2147/OAJSM.S133406] [PMID] [PMCID]

[12] Roper JL, Harding EM, Doerfler D, Dexter JG, Kravitz L, Dufek JS, et al. The effects of gait retraining in runners with patellofemoral pain: A randomized trial. Cli biome. 2016; 35:14-22. [DOI:10.1016/j.clinbiomech.2016.03.010] [PMID]

[13] Liao TC, Powers CM. Tibiofemoral kinematics in the transverse and frontal planes influence the location and magnitude of peak patella cartilage stress: An investigation of runners with and without patellofemoral pain. Clilinical Biomechanics. 2019; 62:72-8. [DOI:10.1016/j.clinbiomech.2019.01.003] [PMID]

[14] Goto S, Aminaka N, Gribble PA. Lower-extremity muscle activity, kinematics, and dynamic postural control in individuals with patellofemoral pain. Journal of Sport Rehabilitation. 2018; 27(6):505-12. [DOI:10.1123/jsr.2016-0100] [PMID]

[15] Naserpour M, Goharpey S, Saki A, Mohammadi Z. Dynamic postural control during step down task in patients with patellofemoral pain syndrome. Journal of Physical Therapy Science. 2018; 30(10):1289-92. [DOI:10.1589/jpts.30.1289] [PMID] [PMCID]

[16] Scholtes SA, Salsich GB. A dynamic valgus index that combines hip and knee angles: Assessment of utility in females with patellofemoral pain. International Journal of Sports Physical Therapy. 2017; 12(3):333-40. [PMID] [PMCID]

[17] de Oliveira Silva D, Briani RV, Pazzinatto MF, Ferrari D, Aragão FA, de Azevedo FM. Reduced knee flexion is a possible cause of increased loading rates in individuals with patellofemoral pain. Clinical Biomechanics. 2015; 30(9):971-5. [DOI:10.1016/j.clinbiomech.2015.06.021] [PMID]

[18] Cunha AB, Babik I, Harbourne R, Cochran NJ, Stankus J, Szucs K, et al. Assessing the validity and reliability of a new video goniometer app for measuring joint angles in adults and children. Archives of Physical Medicine and Rehabilitation. 2020; 101(2):275-82. [DOI:10.1016/j.apmr.2019.07.008] [PMID]

[19] Yakut E, Bayar B, Meriç A, Bayar K, Yakut Y. Reliability and validity of reverse visual analog scale (right to left) in different intensity of pain. The Pain Clinic. 2003; 15(1):1-6. [DOI:10 .1163/156856903321196429]

[20] Yalfani A, Raeisi Z. [Investigate changes distribution of force and center of pressure fluctuations in women with and without patellofemoral pain syndrome in static condition (Persian)]. Journal of Sports Medicine. 2015; 7(1):57-68. https://www.sid.ir/fa/journal/ViewPaper.aspx?ID=247962
[21] Dunn JC, Iversen MD. Interrater reliability of knee muscle forces obtained by hand-held dynamometer from elderly subjects with degenerative back pain. Journal of Geriatric Physical Therapy. 2003; 26(3):23-9. [DOI:10.1519/00139143200312000-00004]

[22] Yalfani A, Raeisi Z. [Comparsion of lower limb strength, $\mathrm{Q}$ angle, varus and valgus knee musclein women with patellofemoral pain syndrome (Persian)]. Contemporary Research in Sport Management. 2013; 23(4):37-127. https:// smms.basu.ac.ir/article_335_580ea5b3dbbf76f87e2 f67a5adddc45e.pdf

[23] de Moura Campos Carvalho-E-Silva AP, Peixoto Leão Almeida G, Oliveira Magalhães M, Renovato França FJ, Vidal Ramos LA, Comachio J, et al. Dynamic postural stability and muscle strength in patellofemoral pain: Is there a correlation?. The Knee. 2016; 23(4):616-21. [DOI:10.1016/j. knee.2016.04.013] [PMID]

[24] Page P. Sensorimotor training: A “global” approach for balance training. Journal of Bodywork and Movement Therapies. 2006; 10(1):77-84. [DOI:10.1016/j.jbmt.2005.04.006]

[25] Ahmed AF. Effect of sensorimotor training on balance in elderly patients with knee osteoarthritis. Journal of Advanced Research. 2011; 2(4):305-11. [DOI:10.1016/j.jare.2011.02.001]

[26] Ahmad I, Noohu MM, Verma S, Singla D, Hussain ME. Effect of sensorimotor training on balance measures and proprioception among middle and older age adults with diabetic peripheral neuropathy. Gait \& Posture. 2019; 74:114-20. [DOI:10.1016/j.gaitpost.2019.08.018] [PMID]

[27] Elshazly FA, Nambi G, Elnegamy TE. Comparative study on virtual reality training (VRT) over sensory motor training (SMT) in unilateral chronic osteoarthritis-A randomized control trial. International Journal of Medical Research Health Science. 2016; 5(8):7-16. https://www.ijmrhs.com/ abstract/comparative-study-on-virtual-reality-training-vrtover-sensory-motor-training-smt-in-unilateral-chronic-osteoarthritis---619.html

[28] Moutzouri M, Coutts F, Gliatis J, Billis E, Tsepis E, Gleeson $\mathrm{N}$. Early initiation of home-based sensori-motor training improves muscle strength, activation and size in patients after knee replacement: a secondary analysis of a controlled clinical trial. BMC Musculoskeletal Disorders. 2019; 20(1):231 [DOI:10.1186/s12891-019-2575-3] [PMID] [PMCID]

[29] McCaskey MA, Wirth B, Schuster-Amft C, de Bruin ED Postural sensorimotor training versus sham exercise in physiotherapy of patients with chronic non-specific low back pain: An exploratory randomised controlled trial. PloS One. 2018; 13(3):e0193358. [DOI:10.1371/journal.pone.0193358] [PMID] [PMCID]

[30] Hwang JA, Bae SH, Do Kim G, Kim KY. The effects of sensorimotor training on anticipatory postural adjustment of the trunk in chronic low back pain patients. Journal of Physical Therapy Science. 2013; 25(9):1189-92. [DOI:10.1589/ jpts.25.1189] [PMID] [PMCID]

[31] Page P, Frank C, Lardner R. Assessment and treatment of muscle imbalance: The Janda approach. Journal of Orthopedic \& Sports Physical Therapy. 2011; 41(10):799-800.

[32] Bruhn S, Kullmann N, Gollhofer A. The effects of a sensorimotor training and a strength training on postural stabilisation, maximum isometric contraction and jump per- 
formance. International Journal of Sports Medicine. 2004; 25(1):56-60. [DOI:10.1055/s-2003-45228] [PMID]

[33] Amal F, Ahmed PF. Effect of sensorimotor training on joint proprioception and isokinetic strength ratios in subjects with unilateral functional ankle instability. Bulletin of Faculty of Physical Therapy. 2010; 15(2):25-34. http://www.lib.pt.cu. edu.eg/4-Amel\%20\%20July\%202010.pdf

[34] Arvidsson I, Eriksson E, Knutsson E, Arnér S. Reduction of pain inhibition on voluntary muscle activation by epidural analgesia. Orthopedics. 1986; 9(10):1415-9. [PMID]

[35] Hopper AJ, Haff EE, Joyce C, Lloyd RS, Haff GG. Neuromuscular training improves lower extremity biomechanics associated with knee injury during landing in 11-13 year old female netball athletes: A randomized control study. Frontiers in Physiology. 2017; 8:883. [DOI:10.3389/ fphys.2017.00883] [PMID] [PMCID]

[36] Barendrecht M, Lezeman HCA, Duysens J, Smits-Engelsman BCM. Neuromuscular training improves knee kinematics, in particular in valgus aligned adolescent team handball players of both sexes. The Journal of Strength \& Conditioning Research. 2011; 25(3):575-84. [DOI:10.1519/ JSC.0b013e3182023bc7] [PMID]

[37] de Almeida Novello A, Garbelotti Jr S, Rabelo NDDA, Ferraz AN, Bley AS, Correa JCF, et al. Descending stairs: Good or bad task to discriminate women with patellofemoral pain? Gait \& Posture. 2018; 65:26-32. [DOI:10.1016/j.gaitpost.2018.06.170] [PMID]

[38] Rathleff MS, Samani A, Olesen JL, Roos EM, Rasmussen S, Madeleine P. Effect of exercise therapy on neuromuscular activity and knee strength in female adolescents with patellofemoral pain-an ancillary analysis of a cluster randomized trial. Clinical Biomechanics. 2016; 34:22-9. [DOI:10.1016/j. clinbiomech.2016.03.002] [PMID]

[39] Powers CM. Rehabilitation of patellofemoral joint disorders: A critical review. Journal of Orthopaedic \& Sports Physical Therapy. 1998; 28(5):345-54. [DOI:10.2519/ jospt.1998.28.5.345] [PMID]

[40] Palmer K, Hebron C, Williams JM. A randomised trial into the effect of an isolated hip abductor strengthening programme and a functional motor control programme on knee kinematics and hip muscle strength. BMC Musculoskeletal Disorders. 2015; 16:105. [DOI:10.1186/s12891-015-0563-9] [PMID] [PMCID]

[41] Ferber R, Kendall KD, Farr L. Changes in knee biomechanics after a hip-abductor strengthening protocol for runners with patellofemoral pain syndrome. Journal of Athletic Training. 2011; 46(2):142-9. [DOI:10.4085/1062-6050-46.2.142] [PMID] [PMCID]

[42] Earl JE. Gluteus medius activity during 3 variations of isometric single-leg stance. Journal of Sport Rehabilitation. 2005; 14(1):1-1. [DOI:10.1123/jsr.14.1.1]

[43] Röijezon U, Clark NC, Treleaven J. Proprioception in musculoskeletal rehabilitation. Part 1: Basic science and principles of assessment and clinical interventions. Manual Therapy. 2015; 20(3):368-77. [DOI:10.1016/j.math.2015.01.008] [PMID]

[44] Prentice WE. Rehabilitation techniques in sports medicine. Dubuque: WCB/McGraw-Hill; 2015. 
This Page Intentionally Left Blank 